\title{
A RETIRADA DOS URÓLITOS DE OXALATO DE CÁLCIO E DESOBSTRUÇÃO DA URETRA ATRAVÉS DA REALIZAÇÃO DA TÉCNICA CIRÚRGICA URETROSTOMIA EM CÃES: RELATO DE CASO
}

\author{
Silvia Rayner Rodrigues de Almeida \\ Johnny Iglesias Mendes Araujo ${ }^{2}$ \\ Siluana Benvindo Ferreira ${ }^{3}$
}

\begin{abstract}
ALMEIDA, S. R. R. de; ARAUJO, J. I. M.; FERREIRA, S. B. A retirada dos urólitos de oxalato de cálcio e desobstrução da uretra através da realização da técnica cirúrgica uretrostomia em cães: relato de caso. Arq. Ciênc. Vet. Zool. UNIPAR, Umuarama, v. 20, n. 3, p. 173-178, jul./set. 2017.
\end{abstract}

\begin{abstract}
RESUMO: A uretra do macho é uma continuação do sistema de ductos, originada de um óstio interno no colo da bexiga urinária e estendida até o orifício peniano uretral externo na extremidade livre do pênis. Relata-se um caso de um canino, macho, SRD, de três anos, pesando 12 kg. Foi atendido no Hospital Veterinário Universitário "Jeremias Pereira da Silva” - HVU/ UFPI, na área de Clínica Médica e Cirúrgica de cães e gatos. O mesmo apresentava manifestações clínicas como: dificuldade de locomoção, disúria, inapetência, hematúria, anúria, agressividade, dor abdominal, apatia e emagrecimento progressivo. A conduta clínica adotada diante do caso, após o resultado dos exames complementares que confirmaram o diagnostico inicial, indicativo de obstrução uretral total por urólitos de oxalato de cálcio, devido ao insucesso das tentativas clínicas de desobstrução foi optar pela técnica cirúrgica uretrostomia. Decorrido dez dias da cirurgia, o animal retornou para retirada dos pontos, apresentando-se em bom estado, alimentando-se bem, fluxo urinário constante (sem sangue), e andando normalmente. A urolitíase deve ser diagnosticada e tratada o mais previamente possível, uma vez que a doença se complica no avançar dos sinais clínicos, podendo ocasionar casos mais complexos, como obstruções urinárias severas e até morte do animal.
\end{abstract}

PALAVRAS-CHAVE: Estenose uretral. Exame clínico. Urolitíase.

\section{REMOVAL OF CALCIUM OXALATE UROLITHS AND URETHRAL CLEARING THROUGH URETHROSTOMY IN DOGS: CASE REPORT}

\begin{abstract}
The male urethra is a continuation of the duct system originated from an internal ostium inside the bladder and extends to the external urethral penile hole at the free end of the penis. This is a case study of a 3-year old male mongrel dog weighing $12 \mathrm{~kg}$. The dog was attended at the University Veterinary Hospital "Jeremias Pereira da Silva" - HVU/UFPI in the Clinical and Surgical Practice of dogs and cats. It presented clinical manifestations such as difficulty in walking, dysuria, loss of appetite, hematuria, anuria, aggressiveness, abdominal pain, lethargy and progressive weight loss. An urethrostomy was the clinical approach adopted for the case, after the laboratory tests confirmed the initial diagnosis of total urethral obstruction by calcium oxalate uroliths due to the failure of clinical unblocking attempts. Ten days after the surgery, the animal returned to the clinic to remove the stitches, and presented a good condition, feeding well, with a constant urine flow (no blood), and walking normally. Urolithiasis must be diagnosed and treated as early as possible, since the disease complicates itself with the worsening of the clinical signs, which may result in more complex cases, such as severe urinary obstruction and even the death of the animal.
\end{abstract}

KEYWORDS: Clinical examination. Urethral stricture. Urolithiasis.

\section{LA RETIRADA DE URÓLITOS DE OXALATO DE CALCIO Y DESOBSTRUCCIÓN DE LA URETRAA TRAVÉS DE REALIZACIÓN DE LA TÉCNICA QUIRÚRGICA URESTROSTOMÍA EN PERROS: RELATO DE CASO}

RESUMEN: La uretra del macho es una continuación del sistema de ductos, originada de un ostium interno en el cuello de la vejiga urinaria y se extiende hasta el orificio uretral externo en la extremidad libre del pene. Presentamos un caso de un canino, macho, SRD, de tres años, con un peso de $12 \mathrm{~kg}$. Fue atendido en el Hospital Veterinario de la Universidad "Jeremías Pereira da Silva" - HVU/UFPI, en el campo de Clínica Médica y Quirúrgica de perros y gatos. Lo mismo presentaba manifestaciones clínicas como: dificultad para caminar, disuria, pérdida de apetito, hematuria, anuria, agresividad, dolor abdominal, letargo y pérdida progresiva de peso. La conducta clínica adoptada delante el caso, tras el resultado de los exámenes complementarios

\footnotetext{
DOI: 10.25110 /arqvet.v20i3.2017.5796

${ }^{1}$ Bacharel em Medicina Veterinária - Universidade Federal do Piauí - UFPI/CPCE, BR 135, km 3 - Bairro Planalto Horizonte, CEP: 64900-000 - Bom Jesus, Piauí, Brasil. Email: silviarayner@gmail.com

${ }^{2}$ Mestre em Zootecnia (PPGZ) - Universidade Federal do Piauí UFPI/CPCE, BR 135, km 3 - Bairro Planalto Horizonte, CEP: 64900-000 - Bom Jesus, Piauí, Brasil. Email: johnny-iglesias@hotmail.com. Autor para correspondência.

${ }^{3}$ Doutora em Ciência Animal - Universidade Federal do Piauí (UFPI), Bairro Ininga, CEP: 64.049-550 - Teresina, Piauí, Brasil. Email: siluanabf@hotmail. com
} 
que confirmaron el diagnóstico inicial, indicativo de obstrucción uretral total de cálculos de oxalato de calcio, debido al fracaso de las tentativas clínicas de desbloqueo cupo optar por uretrostomía, técnica quirúrgica. Después de diez días de la cirugía el animal volvió para retirada de los puntos de sutura, presentándose en buen estado, alimentándose bien, flujo de orina constante (sin sangre), y caminando normalmente. La urolitiasis debe ser diagnosticada y tratada cuanto antes posible, ya que la enfermedad se complica con el avance de los signos clínicos, pudiendo causar casos más complejos, como obstrucciones urinarias severas e incluso la muerte del animal.

PALABRAS CLAVE: Estenosis uretral. Examen clínico. Urolitiasis.

\section{Introdução}

A uretra do macho é anatomicamente definida como uma continuação do sistema de ductos, originada de um óstio interno no colo da bexiga urinária e estendida até o orifício peniano uretral externo na extremidade livre do pênis. Encontra-se envolvida por tecido adiposo e conjuntivo, estando alojada no assoalho pélvico, com a superfície dorsal relacionando-se ao reto e à glândula prostática (FOSSUM, 2002).

A Urolitíase, ou litíase urinária é caracterizada pela presença de urólitos (cálculos, concreções ou pedras) ao longo do trato urinário (MAXIE; NEWMAN, 2007; OSBORNE et al., 2009). Na maioria dos urólitos encontrados em cães 95\% encontram-se na vesícula urinária ou na uretra, assim somente $5 \%$ são localizados nos rins ou nos ureteres (GRAUER, 2010; ARIZA, 2012; INKELMANN et al., 2012).

Os urólitos são classificados e denominados de acordo com a sua composição mineral (OYAFUSO, 2008; OYAFUSO, 2010). Sua formação e composição não têm uma causa isolada, e podem ser influenciadas por diversos fatores incluindo raça, sexo, idade, dieta, anormalidades anatômicas, anormalidades metabólicas, infecção do trato urinário, medicações e pH da urina (LULICH et al., 2004; MOORE, 2007; KOEHLER et al., 2009). Os tipos de urólitos mais comumente encontrados em cães são os de fosfato amoníaco magnesiano (estruvita) e oxalato de cálcio (SOUSA, 2008; OYAFUSO, 2008).

As patologias causadas por urólitos estão entre os problemas mais importantes do trato urinário dos animais domésticos e podem culminar em morte (MAXIE; NEWMAN, 2007; NEWMAN et al., 2007). Extensas lesões secundárias podem formar-se rapidamente no sistema urinário, principalmente se os cálculos causarem obstrução parcial ou completa do fluxo urinário. Entretanto, urólitos não obstrutivos podem persistir por longo tempo sem causar qualquer tipo de lesão nem serem percebidos clinicamente (NETA; MUNHOZ 2008; MAXIE; NEWMAN, 2007; NEWMAN et al., 2007).

Se ocorrer obstrução das vias do trato urinário, a afecção é denominada urolitíase obstrutiva, ocasionando o acúmulo de urina na vesícula urinária, produzindo uma série de complicações como processo inflamatório (cistite), hidronefrose e uremia pós-renal, levando o animal a entrar em um quadro de insuficiência renal aguda (IRA) (GRAUER, 2010; STURION et al., 2011).

A urolitíase é classificada como a terceira doença de maior incidência do trato urinário de cães e gatos. Afeta cerca de $1,5 \%$ a 3,0\% de todos os cães admitidos em clínicas veterinárias e mais de $25 \%$ dos gatos com doença do trato urinário inferior, representando um dos principais motivos de procura ao atendimento clínico emergencial nas clínicas veterinárias (STURION et al., 2011; ARIZA, 2012).

Olsen, (2004) cita que as raças mais predispostas são Schnauzers miniaturas, ShihTzus, Lhasa Apsos, Yorkshire Terriers, Pugs, Dálmatas e Basset Hounds.

Os aspectos clínicos dependem do número, do tipo e da localização dos cálculos no trato urinário, podendo provocar desde anúria, estrangúria, azotemia, disúria, hematúria, dor abdominal e distensão da vesícula urinária (FOSSUM, 2008; GASTIM, 2010; GRAUER, 2010; OLIVEIRA, 2010).

O diagnóstico é baseado no histórico clínico do paciente (anamnese), sinais clínicos, exame físico completo (palpação, cateterismo uretral), exames complementares de diagnóstico por imagem tais como, radiografias simples ultrassonografia abdominal, exames laboratoriais complementares (Hemograma, Urinalise), podendo ocorrer também à eliminação dos urólitos espontaneamente durante a micção (FOSSUM, 2008; OYAFUSO, 2008; GRAUER, 2010; DIAS; SILVA, 2011).

O exame físico é primordial para auxiliar a identificar os problemas que podem predispor o animal à formação de cálculos ou que podem limitar opções terapêuticas. É essencial a realização da palpação abdominal, podendo ser possível diagnosticar cálculos maiores presentes na vesícula urinária e uretra, respectivamente (OLIVEIRA, 2010). Pode-se observar em casos de obstrução uretral total, uma bexiga distendida, túrgida e dolorida durante a palpação (SOUSA, 2008). O prognóstico é reservado, pois não é possível prever a probabilidade de recidiva da urolitíase em seguida ao tratamento clínico ou cirúrgico (BARDELA et al., 2007).

O tratamento da urolitíase pode ser clínico ou cirúrgico, sendo que o tratamento clínico tem como objetivo a dissolução e interrupção do crescimento subsequente dos urólitos (SOUSA, 2008). As desvantagens do tratamento médico incluem o custo que é elevado comparado ao custo do procedimento cirúrgico, devido as múltiplas urinálises, culturas bacterianas e radiografias solicitadas durante o tratamento, e do alto comprometimento do proprietário com o tratamento durante várias semanas ou meses (MONFERDINI; OLIVEIRA, 2010; GRAUER, 2010).

Em condições apropriadas, a cirurgia é o tratamento preferível, pois auxilia a identificação do tipo de urólito e providencia uma terapia médica com uma dieta terapêutica apropriada, evitando recidivas (LULICH et al., 2004). Segundo os mesmos autores a cirurgia deve ser considerada em pacientes com obstrução total do fluxo urinário que não possa ser corrigido por manobras clínicas. Sendo assim, o objetivo desse trabalho é descrever um relato de caso clínico de um canino, macho, SRD, com obstrução urinária causada pela presença de urólitos de oxalato de cálcio na uretra pélvica.

\section{Relato de Caso}

Um canino, macho, SRD, de três anos, pesando12 $\mathrm{kg}$, foi atendido no Hospital Veterinário Universitário "Jeremias Pereira da Silva" - HVU, pertencente à Universidade 
Federal do Piauí - UFPI (Estado do Piauí-PI), na área de Clínica Médica e Cirúrgica de cães e gatos, no período correspondente ao dia 02 de dezembro de 2013 a 02 de janeiro de 2014.

Segundo a proprietária já havia três dias que o animal apresentava manifestações clínicas como: dificuldade de locomoção, disúria, inapetência, hematúria, anúria, agressividade, dor abdominal, apatia e emagrecimento progressivo. $\mathrm{O}$ animal havia sido vermífugado recentemente e a vacinação encontrava-se em dia. A dieta do animal era baseada em ração comercial e comida caseira.

No intuito de sedar o mesmo para possibilitar a realização do exame físico o animal foi submetido a administração de morfina na dose de $0,5 \mathrm{mg} / \mathrm{kg}$ (IM), pois apresentava-se muito inquieto e agressivo devido ao estresse e as fortes dores abdominais.

Durante a avaliação, o animal apresenta-se com atitude apática, estado nutricional bom, temperatura $39,4^{\circ} \mathrm{C}$, leve desidratação, tempo de preenchimento capilar menor do que dois segundos, mucosas normocoradas, linfonodos levemente aumentados, presença de carrapatos. Na palpação abdominal constatou-se distensão de bexiga e dor abdominal ao toque.

Os exames complementares solicitados foram: hemograma, sumário de urina, bioquímico sérico, e radiografia abdominal.

Foi coletado sangue para realização do hemograma, e avaliação da função renal e hepática, assim como sumário de urina. Na avaliação do hemograma não foi observado nenhuma alteração significativa. No entanto o exame bioquímico revelou uma concentração de ureia de $68 \mathrm{mg} / \mathrm{dL}$ valor acima do limite considerado dentro da normalidade para cães que é de 15 - $40 \mathrm{mg} / \mathrm{dL}$. A creatinina com 1,8 mg/dL também encontrava-se acima do valor de referência que é $0,5-1,5 \mathrm{mg} /$ dL para cães, evidenciando uma disfunção renal. O sumário de urina apresentou os seguintes resultados $\mathrm{pH}$ 6,0, além da presença discreta de urólitos de oxalato de cálcio.

O exame radiográfico abdominal revelou a presença de urólitos, que estavam obstruindo a passagem de urina pela uretra do animal (Figura 1).

Figura 1: Exame radiográfico de canino macho, SRD, de três anos de idade com cálculo uretral de oxalato de cálcio, obstruindo uretra.

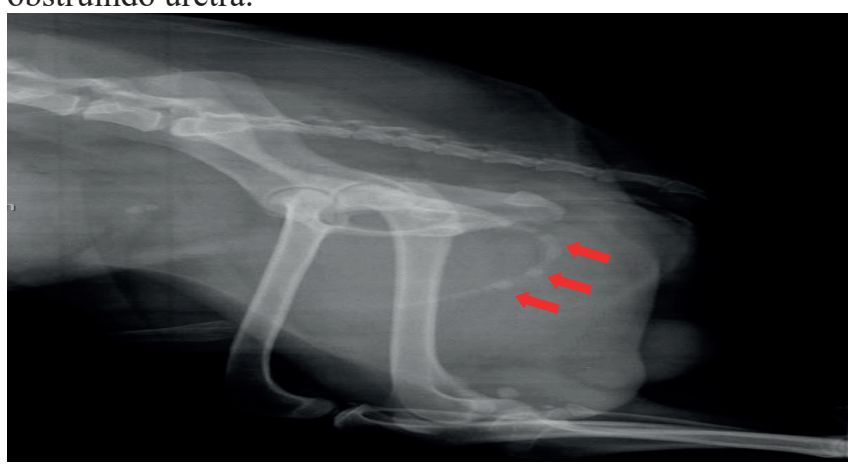

Fonte: HVU - Teresina, Piauí.

O animal encontrava-se em estado crítico, o qual foi constatado durante o exame clínico. Tentou-se a desobstrução por meio da realização de manobra de cateterismo uretral, com o auxílio de sonda $\mathrm{N}^{\circ} 4$, porém sem sucesso, optou- -se por realizar em caráter de emergência uma cistocentese para retirada de urina da bexiga, pois a mesma encontrava-se muito distendida, devido à grande quantidade de líquido nela presente (Figura 2).

Figura 2: A - Realização de cateterismo uretral. B - Cistocentese em canino macho, SRD, de três anos de idade.

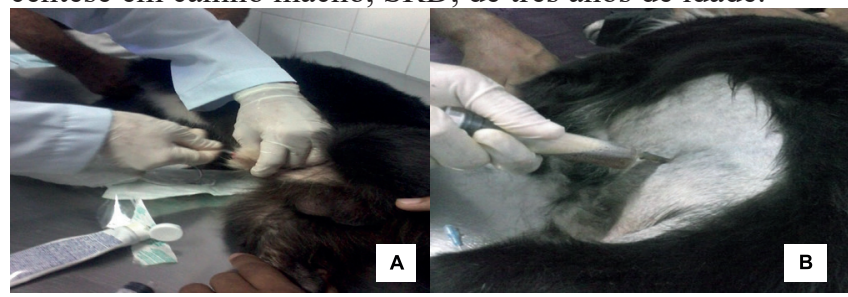

Fonte: HVU - Teresina, Piauí.

A conduta clínica adotada diante do caso, após o resultado dos exames complementares que confirmaram o diagnóstico inicial, indicativo de obstrução uretral total por urólitos de oxalato de cálcio, devido ao insucesso das tentativas clínicas de desobstrução foi optar pela técnica cirúrgica uretrostomia.

O animal ficou internado no HVU em observação, com fluidoterapia solução ringer com lactato por via (EV) na dose de manutenção, até o dia seguinte quando foi encaminhado ao centro cirúrgico do HVU para a retirada dos urólitos de oxalato de cálcio e desobstrução da uretra através da realização da técnica cirúrgica uretrostomia. O animal foi devidamente preparado e encaminhado à sala cirúrgica para a realização da cirurgia de uretrostomia que consistiu: incisão na linha média sobre a uretra, pelo tecido subcutâneo; identificação do músculo retrator peniano, mobilização e retração deste lateralmente para exposição da uretra; incisão da uretra de três a quatro centímetro no interior do lúmen uretral; retirada dos urólitos, aplicação e fixação de sonda uretral na uretra e esvaziamento da bexiga com o auxílio de uma seringa. A fixação da sonda uretral foi feita com o intuito de prevenir estenose durante a cicatrização e limitar a ação da urina sobre a ferida cirúrgica; a finalização foi feita com sutura interrompida simples (Figura 3). No mesmo dia o animal também foi submetido a castração.

A uretrostomia é uma técnica bem sugerida, pois tem um fácil acesso à uretra, o risco de estenose uretral pós-operatório é pequeno e apresenta um prognóstico bom, rápido eficaz. 
Figura 3: A - Animal está sendo preparado para o início da cirurgia. B - A incisão na pele para exposição da uretra do animal. C - A uretra é exposta com o auxílio de uma pinça. D - Uma pequena incisão na própria uretra para inserir a sonda. E - Esvaziamento da bexiga e retirada dos urólitos. F - Sutura da pele, deixando a ponta da sonda para fora.

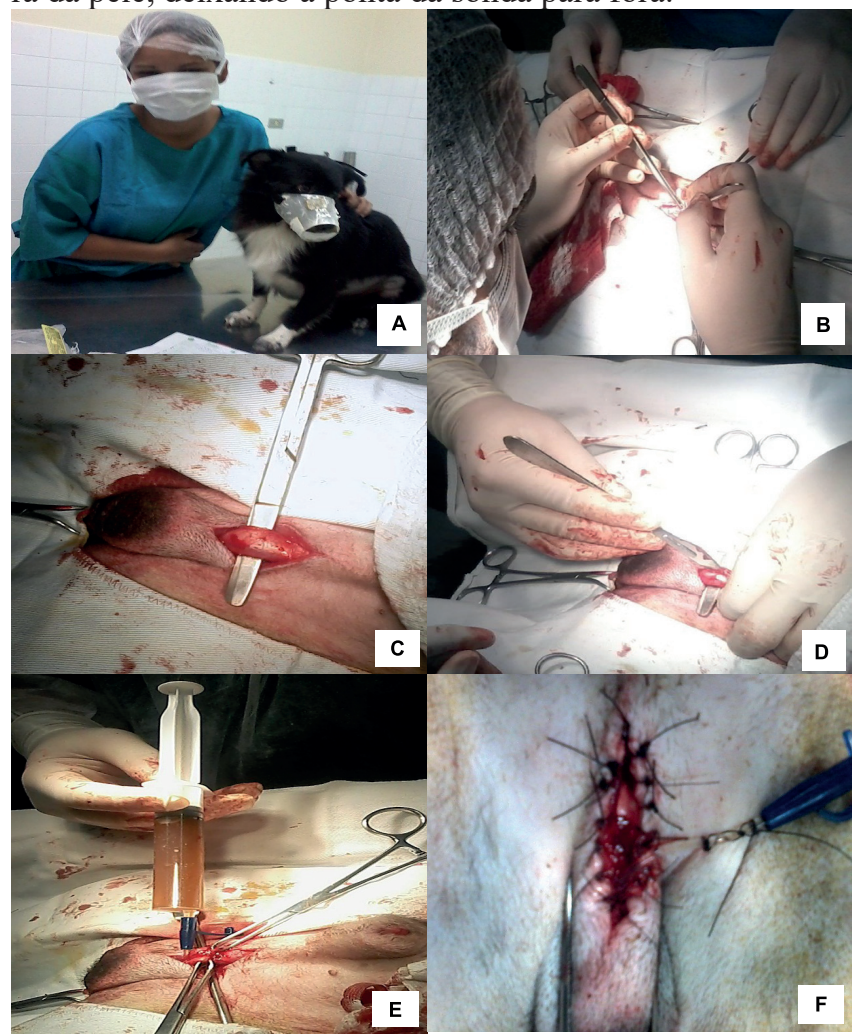

Fonte: HVU - Teresina, Piauí.

Após a cirurgia, o animal permaneceu internado por seis dias, permanecendo sondado por cinco dias. Durante a sua internação foram administrados os seguintes medicamentos: Cefalotina (20 mg/kg/BID/5 dias), cloridrato de tramadol ( $2 \mathrm{mg} / \mathrm{kg} / \mathrm{SID} / 6 \mathrm{dias})$, Morfina $1 \%$ (0,5 mg/kg/SID $/ 5 \mathrm{dias})$, Meloxicam 2\% (0,1- 0,2 mg/kg/SID/3 dias), Glicopangold $®$ (0,5 - 40/ml/SID/5 dias), além da lavagem diária da bexiga urinária com solução fisiologica através da sonda, limpeza da ferida cirúrgica com Rifampcina spray e pomada Nitrofurasona e uso de colar de elisabetano para evitar a lambedura da ferida cirúrgica. No quinto dia foi realizado o exame clínico que constatou a estabilidade do animal permitindo retirada da sonda, pois o animal já urinava e se alimentava normalmente.

Vale destacar que, a cefalotina é um antibiótico do grupo das cefalosporinas da primeira geração, que por sua vez, são classificadas por gerações de acordo com a atividade antimicrobiana (SPINOSA et al., 2006). O Cloridrato de tramadol é um analgésico sintético de ação central, que age em receptores opióides no controle da dor (SASKA et al., 2009), é recomendado para o alívio da dor de intensidade moderada a grave. A Morfina é um fármaco narcótico de alto poder analgésico usado para aliviar dores severas (TRONCY et al. (1996). O Meloxicam é um moderno derivado oxicano que desenvolve uma atividade inibitória seletiva sobre a COX2, na cascata biossintética das prostaglandinas (FROLICH, 1997). O Glicopan gold $\AA$ é um energético rico em nutrientes como nucleotídeos e glutamina, geralmente é indicado como suplemento vitamínico para melhorar a condição nutricional do animal.

No sexto dia após a cirurgia, o animal recebeu alta com prescrição de uma dieta terapêutica. Os alimentos fornecem energia e nutrientes para o animal, sendo que alguns deles são essenciais e devem estar presentes na dieta como os minerais, água, e alguns aminoácidos. A quantidade de cada nutriente a ser oferecida na dieta deve ser adaptada em função do tipo de urólito do animal mantendo o $\mathrm{pH}$ da urina próximo do normal, para prevenir novas recidivas (KONIG et al., 2006). Decorrido dez dias da cirurgia, o animal retornou para retirada dos pontos, apresentando-se em bom estado, alimentando-se bem, fluxo urinário constante (sem sangue), e andando normalmente. Foi realizada a retirada dos pontos, pois a ferida apresentava-se sem inflamação e cicatrizada.

\section{Discussão}

A sintomatologia apresentada pelo animal aliada aos exames complementares, principalmente o radiográfico, confirmou um caso de urolitíase obstrutiva total da uretra. $\mathrm{O}$ animal é um canino, macho, SRD, de três anos de idade. Segundo Picavet et al. (2007) e Rogers (2011) a urolitíase é uma condição clínica importante que é mais recorrente em cães machos que em fêmeas devido às características anatômicas de sua uretra, longa estreita e sinuosa. Podendo ocorrer obstrução principalmente na base do osso peniano (AQUINO et al., 2007). Em alguns casos a obstrução urinária por urólitos leva à ruptura da bexiga e da uretra (MAXIE; NEWMAN, 2007, NEWMAN et al., 2007).

Inkelmann et al. (2012), cita que em um estudo realizado no Rio de Janeiro, onde foram analisadas características epidemiológicas de 76 urólitos de cães encontrados durante a realização de necrópsias no período entre 1990 e 2010. Onde a proporção de machos acometidos por urólitos foi de $64,5 \%$, maior que a de fêmeas, $35,5 \%$. Além de demonstrar que os animais com idade entre um e nove anos foram maioria e representaram $52,6 \%$, comparado com os de mais de 10 anos foram $39,5 \%$ e os com menos de um ano representaram 5,3\%. E os cães sem raça definida representaram $43,4 \%$ dos casos acometidos.

Ainda de acordo com Inkelmann et al. (2012) a alimentação do animal baseava-se em ração comercial e comida caseira com baixa ingestão de água, o que pode ter contribuído para a formação de cálculos no trato urinário inferior do animal. Segundo Carciofi et al. (2007) a composição da dieta pode interferir tanto no aparecimento, quanto na prevenção de recidivas, pois afeta a densidade específica, o volume e o $\mathrm{pH}$ urinário.

Entre os fatores não dietéticos para o aparecimento de urolitíase estão a raça, idade, infecção do trato urinário e sexo. Em cães de pequeno porte, isto ocorre devido a um menor volume urinário excretado e menor número de micções, aumentando assim a concentração de minerais na urina (STEVENSON; RUTGERS, 2006). Segundo Odendaal (1993), a profilaxia possui como base a prevenção do aparecimento dos fatores de risco, bem como o manejo dietético adequado. Assim, fatores diversos, como por exemplo, a ingestão abundante de água limpa e dieta balanceada acabam por auxiliar na prevenção de urólitos em cães, é importante também sempre observar os históricos associados com predisposição familiar. 
Por meio da realização de exames complementares foi possível constatar a presença de urólitos de oxalato de cálcio obstruindo a uretra do animal. Stevenson e Rutgers, (2006) citam que os cálculos de oxalato de cálcio correspondem ao segundo tipo de urólitos mais encontrado em cães e gatos. A nutrição interfere significativamente no aparecimento de urólitos de oxalato de cálcio. Dietas com baixo teor de umidade e sódio, e alta concentração proteica (acidificantes) aumentam os riscos em cães de raças suscetíveis (ELLIOT, 2003).

$\mathrm{O}$ animal apresentou-se $\mathrm{pH}$ urinário 6,0. Segundo Grauer, (2006) a solubilidade do oxalato de cálcio aumenta na urina que apresenta $\mathrm{pH}$ acima de 6,5 , enquanto um $\mathrm{pH}$ entre 4,5 e 6,0 favorece a formação dos cristais de oxalato de cálcio.

O tratamento médico para dissolver urólito por oxalato de cálcio ainda não foi desenvolvido, neste caso recomenda-se a remoção cirúrgica, como foi conduzido nesse caso especifico. Além disso, uma restrição moderada no consumo de proteínas, cálcio, oxalato e sódio, e ingestão normal de fósforo, magnésio, vitamina $\mathrm{D}$ e $\mathrm{C}$, é recomendada para evitar recidiva dos urólitos, após remoção cirúrgica (GRAUER, 2006; OYAFUSO, 2008). A formação de urólitos está intimamente ligada a dieta. Com isso a nutrição quando bem administrada, possui fundamental importância no tratamento e na prevenção das urolitíases, proporcionando melhor qualidade de vida aos animais. O tratamento clínico do animal foi cirúrgico e dietético. Contudo, o proprietário foi informado que o monitoramento deverá ser constante, por que existe a probabilidade de haver recidivas, foi recomendado também avaliações periódicas de exames de urina, bioquímicos, radiografias e ultrassonografia tanto para avaliar a eficácia da dieta como para identificar efeitos colaterais.

\section{Conclusão}

A urolitíase deve ser diagnosticada e tratada o mais previamente possível, uma vez que a doença se complica no avançar dos sinais clínicos, podendo ocasionar casos mais complexos, como obstruções urinárias severas e até morte do animal.

É indispensável a realização de exame clínico e, principalmente exames complementares, buscando por meio destes a apresentação de um diagnóstico preciso. A colaboração do proprietário é essencial ao tratamento do animal, pois os mesmos necessitam de monitoramento e cuidados constantes, assim como o comprometimento do Médico Veterinário em implantar o protocolo de resolução do problema imediato e preventivo a recidivas.

\section{Referências}

AQUINO, L. C.; MARTINS, C. S.; GALERA, P. D. Urolitíase por urato em dálmatas. Revisão de literatura e relato de caso. Clínica Veterinária, v. 12, n. 70, p. 56-66, 2007.

ARIZA, P. C. Epidemiologia da urolitíase de cães e gatos. In: SEMINÁRIOS, 2012. Goiânia. Seminários... 2012, Goiânia: Universidade Federal de Goiás, Goiânia, 2012. 41 f.
BARDELA, G. T. et al. Ruptura de bexiga ocasionada por urolitíase: relato de caso. Revista Científica Eletrônica de Medicina Veterinária, v. 4, n. 8, p. 1-6, 2007.

CARCIOFI, A. C. Métodos para estudo das respostas metabólicas de cães e gatos a diferentes alimentos. Revista Brasileira de Zootecnia, v. 36, suplemento especial, p. 235-249, 2007.

DIAS e SILVA, T. P.; SILVA, F. L. Urolitíase vesical e uretral em um cão: diagnóstico e tratamento. Enciclopédia Biosfera - Centro Científico Conhecer, v. 7, n. 13, p. 970976, 2011.

ELLIOT, D. A. How I treat... the dog with calcium oxalate urolithiasis. Revista Waltham Focus, Buenos Aires, p. 2-3, 2003.

FOSSUM, T. W. Cirurgia da bexiga e da uretra. In: Cirurgia de pequenos animais. 3. ed. Rio de Janeiro: Elsevier, 2008. p. 572-609.

FROLICH, J. C. A classification of NSAIDs according to the relative inhibition of cyclooxygenase isoenzymes. Trends in Pharmacological Sciences, v. 18, n. 1, p. 30-34, 1997.

GRAUER, G. F. Urolitíase canina. In: Medicina interna de pequenos animais. 4. ed. Rio de Janeiro: Elsevier, 2010.

In: Manual de medicina interna

de pequenos animais. 2. ed. Rio de Janeiro: Elsevier, 2006.

GASTIM, T. S. Urolitíase canina. Rio de Janeiro, 2010. 33 f. Monografia (Especialização em clínica médica e cirúrgica de pequenos animais) - Universidade Castelo Branco, São Paulo, 2010.

INKELMANN, M. A. et al. Urolitíase em 76 cães. Pesquisa veterinária brasileira, Rio de Janeiro, v. 32, n. 3, p. 247$253,2012$.

KOEHLER, L. A. et al. Canine uroliths: Frequently asked questions and their answers. Veterinary clinics of north America: Small animal practice. v. 39, n. 1, p. 161-181, 2009.

KONIG, H. E.; MAIERL, J.; LIEBICH, H. G. Órgãos urinários. In: Anatomia dos animais domésticos: texto e atlas colorido. Porto Alegre: Artmed, 2006.

LULICH, J. P.; OSBORNE, C. A.; BARTGES, J. W. Distúrbios do trato urinário inferior dos caninos. In: . Tratado de medicina interna veterinária. 5 . ed. Rio de Janeiro: Guanabara Koogan, 2004. 1841-187.

MAXIE, M. G.; NEWMAN, S. J. The urinary system. In: Palmer's pathology of domestic animals. 5. ed. Philadelphia: Saunders Elsevier, 2007. p. 425-522. 
MONFERDINI, R. P.; OLIVEIRA, J. Manejo nutricional para cães e gatos com urolitíase - revisão bibliográfica. Acta Veterinaria Brasilica, Maringá, v. 3, n. 1, p. 1-4, 2009.

MOORE, A. Quantitative analysis of urinary calculi in dogs and cats. Veterinary Focus, v. 17, n. 1, p. 22-27, 2007.

MUNIZ NETA, E. S.; MUNHOZ, A. D. Urolitíase em cães e gatos: uma revisão. Revista Científica de Medicina Veterinária, v. 17, n. 6, p. 24-34, 2008.

NEWMAN, S. J.; ANTHONY, W. C.; PANCIERA, R. J. Urinary system. In: Pathologic basis of veterinary

disease. 4. ed. Mosby-Elsevier, 2007. p. 613-691.

ODENDAAL, J. Cães e gatos: um guia de saúde. São Paulo: Varela,1993.

OLIVEIRA, A. C. S. Urolitíase canina. Brasília, 2010. 29 f. Monografia (Especialização em Clínica Médica e Cirúrgica de Pequenos Animais) - Universidade Castelo Branco, São Paulo, 2010.

OLSEN, D. Ruptura e cálculos ureterais. In:

Segredos em cirurgia de pequenos animais. Porto Alegre: Artmed, 2004. p. 228-230.

OSBORNE, C. A. et al. Analysis of 451,891 canine uroliths, feline uroliths, and feline urethral plugs from 1981 to 2007 : Perspectives from the Minnesota Urolith Center. Veterinary Clinics of Nort America-Small Animal Pplactice. v. 39, n. 1, p. 183-197, 2009.

OYAFUSO, M. K. Retrospective and prospective study of urolithiasis in dogs. 2008. 146 f. Dissertação (Mestrado em Medicina Veterinária) - Faculdade de Medicina Veterinária e Zootecnia, São Paulo, 2008.

OYAFUSO, M. K. et. al. Urolitíase em cães: avaliação quantitativa da composição mineral de 156 urólitos. Ciência Rural, Santa Maria, v. 40, n. 1, p. 102-108, 2010.

PICAVET, P. et al. Analysis of 4495 canine and feline uroliths in the Benelux. A retrospective study: 1994-2004. Journal of Animal Physiology and Animal Nutrition, v. 91, n. 5, p. 247-251, 2007.

ROGERS, K. D. et al. Composition of uroliths in small domestic animals in the United Kingdom. The Veterinary Journal, v. 188, n. 2, p. 228-230, 2011.

SASKA, S. et al. Cloridrato de tramadol/paracetamol no controle da dor pós-operatória em cirurgias de terceiros molares inclusos. Revista de Cirurgia e Traumatologia Buco-Maxilo-facial. v. 9, n. 4, p. 99-105, 2009.

SOUSA, L. C. Urolitíase canina. Goiânia, 2008. 85 f. Monografia (Especialização em clínica médica e cirúrgica de pequenos animais) - Universidade Castelo Branco, São Paulo, 2008.
SPINOSA, H. S.; GÓRNIAK, S. L.; BERNARDI, M. M. Farmacologia aplicada à medicina veterinária. 4. ed. Rio de Janeiro: Guanabara Koogan, 2006. 469-471 p.

STEVENSON, A.; RUTGERS, C. O. Manejo nutricional de urolitíase canina. In: Enciclopédia de nutrição clínica canina. Paris, 2006.

STURION, D. J. et al. Urolitíase em cães e gatos revisão de litaratura. In: CONGRESSO DE INICIAÇÃO CIENTÍFICA DA FIO, 10., 2011, Ourinhos. Anais... Ourinhos, 2011.

TRONCY, E.; CUVELLIEZ, S. G.; BLAIS, D. Evaluation of analgesia and cardiorespiratory effects of epidurally administered butorphanol in isoflurane-anesthetized dogs. American Journal of Veterinary Research, v. 57, n. 10, p. 1478-1482, 1996.

Recebido em: 04.08.2016. Aceito em: 23.12.2017. 